\title{
REZENSIONEN
}

\section{Große Koalition: Empiriereiche Bilanzen aus Wissenschaft und Journalismus}

Lohse, Eckart und Markus Wehner: Rosenkrieg. Die große Koalition 2005-2009, Fackelträger Verlag, Köln 2009, 272 Seiten, € 16,95.

Egle, Christoph und Reimut Zohlnhöfer (Hrsg.): Die zweite Große Koalition. Eine Bilanz der Regierung Merkel 2005-2009, VS Verlag für Sozialwissenschaften, Wiesbaden 2010, 602 Seiten, $€ 39,95$.

Bukow, Sebastian und Wenke Seemann (Hrsg.): Die Große Koalition. Regierung - Politik - Parteien 2005-2009, VS Verlag für Sozialwissenschaften, Wiesbaden 2010, 391 Seiten, € 34,95.

Die Bundestagswahl 2005 endete für die beiden Volksparteien SPD und CDU/CSU mit einer Enttäuschung. Eine von CDU und CSU angestrebte Koalition mit der FDP war rechnerisch ebenso unmöglich wie ein rot-grünes Bündnis. Neben - damals unwahrscheinlichen - Dreier-Bündnissen blieb nur eine Große Koalition unter Führung der CDU-Bundesvorsitzenden Angela Merkel. Ein gutes Jahr nach Ende dieser zweiten Großen Koalition in der Geschichte der Bundesrepublik Deutschland sind nun die ersten Studien erschienen, die sich systematisch mit ihrer Arbeit und den Politikergebnissen beschäftigen.

Alle drei hier anzuzeigenden Bände gehen implizit der Frage nach, ob es sich bei den vier Jahren der Großen Koalition um eine „Lebensabschnittspartnerschaft“ (Franz Müntefering), um eine „Koalition der neuen Möglichkeiten“ (Angela Merkel) oder um eine „Zweckehe“ (Matthias Platzeck) handelte. Diese zweite Große Koalition war zu Beginn für viele Beobachter, wie Christoph Egle und Reimut Zohlnhöfer in ihrer Einleitung schreiben, "aus der Not heraus geboren“ (S. 11). Sebastian Bukow und Wenke Seemann sekundieren dieser Einschätzung mit den Formeln „Notbehelf in Folge elektoraler Zwänge“ und „Ausnahmeregierung" (S. 15). Auch die Journalisten Eckart Lohse und Markus Wehner teilen diese Einschätzung von der Notlösung: „Hier wird nicht zusammenwachsen, was zusammengehört oder zumindest eine Zeitlang zusammen will, sondern es schlingert aufeinander zu, was alleine untergehen müsste“ (S. 29).

Die Bildung einer Großen Koalition stellt im Bund immer noch eine Ausnahmesituation dar, während es in den vergangenen Jahrzehnten auf Landesebene in jedem fünften Fall einer Regierungsbildung zu einem solchen Bündnis kam (vgl. Bukow / Seemann, S. 9). Die Einschätzungen dieses Koalitionmodells changierten 2005 somit „zwischen der Hoffnung auf grundlegende Reformen im ansonsten politikverflochtenen grand coalition state einerseits und der Annahme einer Gefahr für die Demokratie andererseits" (Bukow / Seemann, S. 9). Im Vergleich zur ersten Großen Koalition von 1966 bis 1969 unter Leitung von Bundeskanzler Kurt Georg Kiesinger fanden sich beide Parteien nun unter ganz anderen Bedingungen zusammen. War es damals eine bewusste Entscheidung beider Partner, ein Bündnis zu schließen, so blieb rein rechnerisch in diesem Fall keine andere ernsthafte Alternative. Vergleiche mit der Großen Koalition in der „alten“ Bundesrepublik, ohne Grüne und Linkspartei, sagten somit „nicht allzu viel über die Chancen und Risiken für eine solche Koalition im Jahr 2005 aus" (Lohse / Wehner, S. 27). 
Die Besonderheit der Großen Koalition unter Bundeskanzlerin Angela Merkel lässt sich anhand einiger Zahlen illustrieren. Im 16. Deutschen Bundestag stand einer Regierungsmehrheit von 73 Prozent der Abgeordneten die oppositionelle Minderheit von 27 Prozent gegenüber (443 zu 165 Sitze, vgl. Seemann, S. 45). Damit vereinigten CDU/CSU und SPD mehr als zwei Drittel der Mitglieder des Bundestages auf sich. Mit CDU/CSU (222 Sitze) und SPD (221 Sitze) standen sich in der Regierungskoalition zwei etwa gleich große Partner gegenüber. Die Opposition stellte mit drei nahezu gleich großen Fraktionen (FDP: 61, Linke: 53, Grüne: 51 Sitze) eine deutlich stärkere Kraft dar als während der ersten Großen Koalition, allerdings war sie in der 16. Wahlperiode deutlich heterogener: Mit diesen Fraktionen standen „erstmals in der Geschichte der Bundesrepublik drei Oppositionsparteien der Bundesregierung gegenüber, die auf Bundesebene vorher noch nie miteinander koaliert haben und höchst unterschiedliche politische Programme verfolgen " (Seemann, S. 45). Lohse und Wehner weisen einleitend auf ein weiteres Spezifikum hin: „Tatsächlich ist das hervorstechende Merkmal dieser Großen Koalition nicht ihre schiere Größe, die Zahl der Bundestagsmandate, die ihr zu einer sicheren Mehrheit im Parlament verhilft. Viel wichtiger ist, dass hier erstmals zwei Parteien eine Bundesregierung miteinander bildeten, die das ausdrücklich nicht gewollt und bis zum Wahlabend zurückgewiesen haben. Es sind zwei Parteien, die im anderen jahrzehntelang den Hauptkonkurrenten gesehen haben" (Lohse / Wehner, S. 8 f.). Diese Einschätzung bleibt nach Meinung der Autoren nicht ohne Konsequenzen für die Arbeit der Regierung: „Es ist nicht so sehr die Sorge, man werde vier Jahre mit den ,anderen' nicht durchhalten. Die Gefahr wäre bei völlig neuen Modellen, bei denen FDP und Grüne zusammengebracht werden müssten oder die Linkspartei Oskar Lafontaines eine Rolle spielte, viel größer. Was Union und SPD fürchten, ist vielmehr der Verlust von Identität und Profil, von Wahrnehmung durch die Wähler und mithin von Zustimmung bei der nächsten Wahl“ (S. 10). Dazu präsentieren Udo Zolleis und Julia Bartz im Band von Egle und Zohlnhöfer die empirischen Ergebnisse (vgl. S. 64 ff.): So hat die Union augenscheinlich stärker von der Großen Koalition profitiert als die Sozialdemokraten - eine Einschätzung, die von Harald Schoen in seiner Analyse der „Einstellungen zur Großen Koalition und Wahlverhalten 2009" bestätigt wird (vgl. S. 45 f.).

Beide Sammelbände aus dem VS Verlag bieten eine Fülle an empirischen Belegen und wissenschaftlichen Analysen. Auf absehbare Zeit dürfte kein Wissenschaftler, der sich mit der Großen Koalition beschäftigt, auf diese beiden Titel verzichten können. Was sie an politikwissenschaftlichen Befunden bieten, ergänzt das Buch von Lohse und Wehner anhand vieler Fallbeispiele und Beobachtungen aus der politischen Praxis. Die beiden Autoren, die für die Frankfurter Allgemeine Sonntagszeitung arbeiten, beschreiben in ihrer Einleitung eindrücklich die Situation, in der sich beide Volksparteien nach der Wahl vom September 2005 wiederfanden. Sie stellen die Motivlagen der einzelnen Akteure detailliert dar und dokumentieren die ersten Schritte hin zur Großen Koalition. Zwangsläufig konzentrieren sie sich auf das Akteurshandeln: Wer geht mit welchen Vorstellungen in die Verhandlungen? Welche Strategien verfolgen die einzelnen in Bezug auf inhaltliche und personelle Weichenstellungen? Warum fallen die Ressorts an die jeweiligen Parteien, welche Politiker kommen zum Zuge?

So zitieren Lohse und Wehner den SPD-Bundestagsabgeordneten Johannes Kahrs, Sprecher des Seeheimer Kreises der SPD, mit den Worten: „Die Frage ist, warum wir die Probleme haben und die Union die Zukunft" (S. 39). Damit spielt er an auf die Ressortverteilung, bei der die zukunftsträchtigen Ressorts wie Bildung, Wirtschaft, Verbraucherschutz und Familie an die CDU und CSU gingen, die schwierigen Ministerien wie Gesundheit, 
Arbeit und Soziales sowie Umwelt an die SPD. Dass die nicht den Bundeskanzler stellende Partei das Außenamt übernimmt, ist inzwischen fast schon eine Tradition, damit der Juniorpartner „die erfahrungsgemäß große Wirkung des Regierungschefs auf der internationalen Bühne wenigstens ein bisschen ausgleichen kann" (S. 37). Hier klingt eine Strategie der CDU-Vorsitzenden Angela Merkel an, nämlich die Arbeit der Regierung bereits zu Beginn auf die nächste Wahl und auf die dann wichtigen Wählergruppen zu fokussieren. Diese Strategie wird in den Beiträgen zum konkreten Regierungsstil Merkels von Karl-Rudolf Korte (im Band von Bukow / Seemann) und Manuela Glaab (im Band von Egle / Zohlnhöfer) systematisch vertieft und erweitert dargestellt. Aufschlussreich ist die Beobachtung von Lohse und Wehner, dass die Unionsseite bewusst Ressorts auswählte, deren Zielgruppen der Union bei der nächsten Bundestagswahl insofern helfen können, als dann eine „kleine“ Koalition mit der FDP möglich sein sollte: „Schon bei der Zusammenstellung des Kabinetts zeigt sich also ein entscheidendes Konstruktionsproblem, das die Große Koalition in den nächsten Jahren begleiten wird. Noch bevor Angela Merkel Kanzlerin ist, treibt sie der Gedanke, wie sie bei der nächsten Bundestagswahl besser abschneiden könne, mehr um als die Überlegungen, wie die Reform des Sozialstaats fortgesetzt wird, das Steuersystem neu geordnet oder das Gesundheitswesen umstrukturiert werden kann" (Lohse / Wehner, S. 39). Entsprechend schwierig war die Eingewöhnungszeit der Akteure dieser unterschiedlichen Partner. Kennzeichnend dafür ist etwa der von Lohse und Wehner kolportierte Ausspruch von Justizministerin Brigitte Zypries nach der ersten Ministerrunde Ende 2005 in Genshagen bei Berlin: Es sei ein bisschen so gewesen, „wie wenn sich Stachelschweine lieben“ (S. 51).

Das Manuskript für „Rosenkrieg“ wurde im Dezember 2008 abgeschlossen. Damit konnten die Anfänge der Wirtschafts- und Finanzkrise im Herbst 2008 zwar noch mit abgedeckt werden, der Zeitraum bis zur Neuwahl im September 2009 aber leider nicht mehr berücksichtigt werden. Dabei wäre es gerade auch aus journalistischer Sicht interessant gewesen zu erfahren, inwiefern - und wie - der nahende Wahlkampf und die anstehende Bundestagswahl 2009 die Arbeit der Großen Koalition beeinflusst haben. Laut mehrerer Beiträge der vorgestellten Sammelbände war dies einer der zentralen Gründe, warum die Große Koalition am Ende ihrer Regierungszeit an Effizienz und Effektivität verlor.

Profitiert hat die Arbeit der Koalition sicher auch von der Tatsache, dass die meisten Minister vor Beginn der Großen Koalition Erfahrungen in der Exekutive sammeln konnten (vgl. Lohse / Wehner, S. 41). Bezeichnend ist auf der anderen Seite, dass nicht ein einziger aktiver Ministerpräsident der Union zum Regieren nach Berlin kam (vgl. Lohse / Wehner, S. 41). Mehr noch: der bayerische Ministerpräsident Edmund Stoiber sprach Merkel öffentlich das Weisungsrecht ab und verkündete, die wichtigen Entscheidungen würden im Koalitionsausschuss der Partei- und Fraktionsvorsitzenden getroffen werden (Lohse / Wehner, S. 42). Damit stellte sich bereits zum Zeitpunkt der Koalitionsverhandlungen die Frage nach der Richtlinienkompetenz der Bundeskanzlerin in einem Bündnis nahezu gleich großer Partner. Der damalige Parteivorsitzende der SPD, Franz Müntefering, beschrieb das Kanzlerprinzip als „nicht lebenswirklich“ (Lohse / Wehner, S. 42).

In mehrseitigen Kurzporträts werden bei Lohse und Wehner mit Angela Merkel, FrankWalter Steinmeier, Franz Müntefering, Edmund Stoiber, Wolfgang Schäuble und Oskar Lafontaine die einzelnen Akteure lebendig. Ihre Handlungsmotive werden beleuchtet und ihre Rolle im politischen Wirken der Koalition nachgezeichnet. Insgesamt eignet sich das Werk von Wehner und Lohse sehr gut zur Ergänzung der beiden politikwissenschaftlichen Sammelbände. 
Bukow und Seemann setzen drei Schwerpunkte: Im ersten Teil findet - nach einer umfassenden Einführung durch die Herausgeber selbst - eine Annäherung an die Arbeit der Großen Koalition in systematischer Perspektive statt. Wenke Seemann blickt auf die Gesetzgebungsarbeit der Koalition zurück. Insbesondere fragt sie nach den Unterschieden zwischen dem seit der Vereinigung Deutschlands vermehrt auftretenden „Grand Coalition State", also der faktischen informellen Koalition, und der Bildung einer formellen Großen Koalition (vgl. S. 43 f.). Seemann identifiziert die „spezifischen Interaktionsmuster von Union und SPD, Regierung und Opposition im Bundestag sowie von Bundesregierung und Bundesrat unter der Bedingung der Großen Koalition“ (Seemann, S. 44).

Astrid Lorenz fragt daran anknüpfend unter der Überschrift „Schutz vor der Mehrheitstyrannei?" nach den potentiellen Kontrolleuren der Zweidrittelmehrheit von CDU/CSU und SPD im Bundestag und konzentriert sich dabei auf die parlamentarische Opposition, das Bundesverfassungsgericht und den Bundespräsidenten. Das Besondere an Großen Koalitionen ist ihrer Einschätzung nach, dass „die Regierungseinbindung der beiden stärksten Parteien institutionelle Barrieren außer Kraft setzt, die sich die Verfassungsgeber ausgedacht haben, um die Machtinhaber zu zügeln“ (Lorenz, S. 59). Während ihrer Untersuchung nach das Bundesverfassungsgericht ohne besondere Auffälligkeiten seinem Kontrollauftrag nachkam, erwies sich der Bundespräsident in Person von Horst Köhler als - allerdings nur punktuell wirkender - „nachträglicher Vetospieler“ (S. 80). In Bezug auf die parlamentarische Opposition bilanziert Lorenz ein ambivalentes Ergebnis: Die „Instrumente sanfter Kontrolle“ (S. 81) wurden demnach vermehrt angewandt. Die Zahl der Kleinen Anfragen stieg ebenso an wie die Zahl der namentlichen Abstimmungen. Dabei agierten die Oppositionsfraktionen überwiegend separat, selbst bei „strukturell ähnlichen Präferenzen“ (S. 81). Andere Parameter der parlamentarischen Oppositionsarbeit blieben im Vergleich zu den vorangegangenen Wahlperioden nahezu unverändert.

Werner Reutter beleuchtet in seinem Beitrag die Arbeit der Großen Koalition an der Föderalismusreform, die mit 25 (von 192) betroffenen Artikeln (einschließlich Präambel und Anhang) die größte Grundgesetzreform in der Geschichte der Bundesrepublik war (S. 86). Bei dieser „Mutter aller Reformen“ (Edmund Stoiber, zitiert in Reutter, S. 91) handelte es sich deshalb um ein zentrales Projekt, weil zum einen die „seit der Vereinigung gestiegene ökonomische, soziale und politische Heterogenisierung der Länder bundeseinheitliche Lösungen erschwerte und die Entscheidungsfindung im Bund verkomplizierte" (S. 87); gleichzeitig stieg die Zahl der zustimmungsbedürftigen Gesetze nach 1990 sukzessive auf rund 60 Prozent (Details vgl. S. 86).

Karl-Rudolf Korte widmet sich unter der Überschrift „Präsidentielles Zaudern“ dem Regierungsstil von Bundeskanzlerin Angela Merkel von 2005 bis 2009. Er untersucht ihn anhand des "Steuerungstrilemmas“ (S. 105) und dessen drei Ebenen: der Parteiendemokratie, der Verhandlungsdemokratie sowie der Mediendemokratie (vgl. S. 104 f.). Was Angela Merkel als Kanzlerin einer Großen Koalition an Handlungsautonomie einbüßte, versuchte sie, „über den Schein einer Politik der Kohärenz spielerisch als Autonomiegewinn für sich zu verbuchen“ (S. 106 f.). Korte sieht auch einen potentiellen Nachteil des Merkelschen Stils: Praktiziert sie vor allem „Dissensmanagement“ (S. 107), kann dies Stammwähler verunsichern. „Kohärenz kann dann nach Jahren des Regierens auch Profillosigkeit zur Folge haben [...] Der Kanzlerbonus ist in der Großen Koalition nur erreichbar um den Preis unklarer Parteibotschaft“ (ebenda). Als weitere Merkmale charakterisiert Korte Machtzentralisierung (Ausbau der eigenen Parteimacht, das Kanzleramt als „Präsidial-Büro“), stilles 
Regieren (Informalisierung, Koalitionsausschuss als Entscheidungszentraum der Regierung), Netzwerk-Pflege, Chefsachen-Mythos, Telepolitik (ausgeprägtes Medienregime der Kanzlerin), Policy-Akzentuierung (Außenpolitik, später auch durch die globale Wirtschaftsund Finanzkrise) und Ideen-Management (das Markenzeichen einer Kanzlerschaft). All diesen Techniken ist laut Korte eines gemeinsam: Sie sollen den Akteuren Entscheidungskompetenz und Steuerungspotentiale sichern beziehungsweise zurückgewinnen helfen.

Ergänzend zu Korte lässt sich auf den Beitrag von Glaab im Band von Egle und Zohlnhöfer zurückgreifen, der das Thema aus einer anderen Perspektive betrachtet. Er folgt einem „interaktionistischen Verständnis politischer Führung" (S. 124) und untersucht drei komplementäre Funktionsbereiche politischer Führung: die Definition der Regierungsagenda; effiziente Steuerung und Koordination des Regierungsapparats; das Informations- und Interdependenzmanagement in Netzwerken sowie die „Allegiance Roles“, die auf den Mehrheitserhalt und die Mobilisierung öffentlicher Unterstützung abzielen (vgl. S. 123 ff.). Einig sind sich Korte und Glaab, dass in einer Großen Koalition nicht die Bestimmungsfaktoren der Kanzlerdemokratie nach Art. 65 GG zum Tragen kommen können, sondern eher die „Inszenierung der Nichtinszenierung“ und „mühsames Patchwork“ (Korte, S. 102) sowie die „kollegiale Sachverwalterin des Übergangs“ mit einem „adaptiv-moderierend[en]“ Regierungsstil (Glaab, S. 151): „Als präsidiale Vermittlerin kann sie unter den Bedingungen einer Großen Koalition nicht kraftvoll führen, selbst wenn sie es wollte. Ausgleichen, lavieren, taktieren, schlichten und moderieren gehören zum Entscheidungsstil einer Kanzlerin der Großen Koalition“ (Korte, S. 103).

Nach diesen Polity- und Politics-zentrierten Abschnitten folgt im Band von Bukow und Seemann die Untersuchung einer Reihe von Politikfeldern. Hier werden sieben skizziert, während im Band von Egle und Zohlnhöfer mit 17 Politikbereichen der Bogen weiter gespannt wird (zum Beispiel auch die Pflege-, die Verfassungs- und die Verwaltungspolitik, die Außen- und Sicherheitspolitik sowie die Europapolitik). Hier identifiziert Frank Wendler vor allem drei Elemente: eine Haltung des interessegeleiteten Pragmatismus, starke politische Führungsleistungen der Akteure und Anzeichen einer allmählichen Erosion des europapolitischen Konsenses im Bundestag (vgl. S. 531). In vielen Bereichen, dies zeigen gleich mehrere Beiträge in den beiden Sammelbänden, hat sich die Koalition auf „die bei der Atomstromerzeugung angewandte Methode, sich wegen unüberbrückbarer Differenzen aufs Nichthandeln zu einigen" (Lohse / Wehner, S. 10) beschränkt. So sollte der Nachfolgerregierung - wie auch immer sie aussehen würde - die Möglichkeit gegeben werden, eigene Vorstellungen konsequent umzusetzen.

Am Ende kann sich die inhaltliche Bilanz dieser ungewollten Partnerschaft zweier erklärter Gegner durchaus sehen lassen. Es handelte sich nach Meinung von Wehner und Lohse zwar nicht um eine „Wohlfühl-Koalition“ (S. 51), dennoch fällt die inhaltliche Bilanz der Regierung nicht zwangsläufig bescheiden aus. In beiden Sammelbänden wird der Policy-Output der Großen Koalition differenziert betrachtet. Während einige wichtige Erfolge möglich waren, fällt die Bilanz in anderen Bereichen weniger überzeugend aus. Positiv bewertet werden etwa mit Blick auf innenpolitische Themen (mit Einschränkungen) die Föderalismusreform, die Familienpolitik, die Umweltpolitik (zumindest in den ersten Jahren), die Integrationspolitik, die Unternehmenssteuerreform und die Haushaltskonsolidierung, bevor der Ausbruch der Wirtschafts- und Finanzkrise diese zur Makulatur werden ließ. Außenpolitisch erhalten die Nahostpolitik, die Energiesicherungspolitik, die Klimapolitik sowie die Verhandlungsführung der Bundesregierung bei der Revision des europäischen 
Verfassungsvertrages gute Noten. Kritisch werden die Bemühungen um das gescheiterte Umweltgesetzbuch gesehen, ebenso die beiden zentralen Privatisierungsprojekte der 16. Wahlperiode (Bahn, Flugsicherung) und die Reaktion auf das Urteil des Bundesverfassungsgerichts zur Mischverwaltung durch Arbeitsgemeinschaften von Arbeitsagenturen und Kommunen (vgl. Egle / Zohlnhöfer, S. 579 ff.). Einige Politikfelder wie etwa die Wirtschafts- und Finanzpolitik sowie der Umgang mit der globalen Krise in den Jahren 2008 und 2009 werden wohl erst in einiger Zeit angemessen bewertet werden können.

In einem dritten Abschnitt des Bandes von Bukow und Seemann wird nach dem Parteienwettbewerb und der Parteienentwicklung unter den Bedingungen der Großen Koalition gefragt. Zunächst gibt Oskar Niedermayer einen breiten und generellen Überblick über „Parteien und Parteiensystem“. Anschließend werden die einzelnen Parteien sowie deren Handeln in der Zeit der Großen Koalition in Einzelbeiträgen beleuchtet. Wolfgang Schroeder und Arijana Neumann schreiben über die CDU, Heinrich Oberreuter erörtert die „Erosion der CSU“ während der Großen Koalition. Uwe Jun fragt in seinem Beitrag über die SPD, ob die Partei in ihrer Zeit und Funktion als Juniorpartner der Großen Koalition einen selbstverschuldeten Niedergang zu verantworten hat oder ob es sich hier um eine zwanghafte Anpassung an veränderte Ausgangsbedingungen der Politik handelt. Die Oppositionsparteien werden in einem gemeinsamen Kapitel (Jens Walther: „Zwischen Kooperation und Blockade") behandelt.

Diese Zusammenhänge finden sich in ähnlicher Form auch im Band von Egle und Zohlnhöfer. Beide Koalitionspartner werden ausführlich berücksichtigt - die Union von Zolleis und Bartz („Unbestimmt erfolgreich“), die SPD von Joachim Raschke („Zerfallsphase des Schröder-Zyklus“). Egle ergänzt diesen Teil mit einer Gesamtschau des Parteienwettbewerbs während dieser 16. Wahlperiode des Bundestages („Im Schatten der Linkspartei“). Insgesamt ist dieser Hauptteil bei Egle und Zohlnhöfer deutlich knapper ausgefallen.

Der dritte Teil des von Bukow und Seemann herausgegebenen Bandes wird abgeschlossen durch einen Beitrag von Stefan Hunsicker und Yvonne Schroth, die sich mit der Sicht des Wählers auf die Große Koalition beschäftigen. Sebastian Scharch fügt eine ausführliche, fast dreißig Seiten umfassende, nützliche Chronologie der Arbeit der Großen Koalition hinzu. Leider findet sich in diesem Band kein bilanzierendes Abschlusskapitel - wie es Egle und Zohlnhöfer beispielhaft machen und dort auf ihre eingangs aufgeworfene Frage nach der Reform- oder Blockadethese rekurrieren: Bedarf es einer solchen Koalition zweier großer Partner, um wesentliche Reformen durchsetzen zu können, oder blockieren sich beide Parteien, da sie sich nur auf den „kleinsten gemeinsamen Nenner“ (S. 20) einigen können (vgl. S. 17 ff., S. 578 ff.)?

Beiden Sammelbänden gelingt es in vorzüglicher Weise, nicht nur einen breiten Überblick über die Arbeit der Großen Koalition zu geben, sondern - so Bukow und Seemann in ihrer Vorbemerkung - auch zu beschreiben und zu erörtern, ob und inwieweit dieses Regierungsbündnis das Land und das politische System verändert hat: „Es ist schließlich anzunehmen, dass bis zur nächsten Großen Koalition im Bund nicht erneut fast vierzig Jahre ins Land gehen werden“ (S. 7). Leider sind viele Beiträge vor der Bundestagswahl 2009 abgeschlossen worden, das Manuskript des Buches von Lohse und Wehner sogar bereits im Dezember 2008. Hier hätte es sich als interessant und lohnenswert erwiesen, die Geschichte der Großen Koalition bis zum Tag der Bundestagswahl am 27. September 2009 weiterzuführen. 